\title{
Impact of correlator efficiency errors on SMOS land-sea contamination
}

\author{
Ignasi Corbella, Israel Duran, Student Member, IEEE, Lin Wu, Student Member, IEEE, \\ Francesc Torres, Senior Member, IEEE, Nuria Duffo, Senior Member, IEEE, Ali Khazâal, and \\ Manuel Martín-Neira, Senior Member, IEEE
}

\begin{abstract}
Land-sea contamination observed in SMOS (Soil Moisture and Ocean Salinity) brightness temperature images is found to have two main contributions: the floor error inherent of image reconstruction and a multiplicative error either in the antenna temperature or in the visibility samples measured by the correlator. The origin of this last one is traced down to SMOS calibration parameters to yield a simple correction scheme which is validated against several geophysical scenarios. Auto-consistency rules in interferometric synthesis together with redundant and complementary calibration procedures provide a robust SMOS calibration scheme.
\end{abstract}

Index Terms-Radiometry, interferometry, imaging, calibration, SMOS.

\section{INTRODUCTION}

Since its launch in November 2009, the European Space Agency (ESA) SMOS mission [1] has provided a large amount of valuable and consolidated geophysical data over land, ice and ocean [2]. Since there is still room for refinement, SMOS mission operation relies on "Expert Support Laboratories" (ESL) responsible of defining new algorithms and methodologies to further improve the quality of SMOS products. This activity is crystallized in the periodic development of data processor versions at different levels. For example, the latest Level-1 operational processor (L1OP v620) will become nominal in spring 2015 and has been used in the second mission reprocessing completed in September 2014, although not yet available to the users. Among other calibration enhancements, this version includes a fully polarimetric image reconstruction scheme [3]. Preliminary analysis of the reprocessed data confirms an important improvement in the data quality, especially in the third and fourth Stokes parameters. The work presented in this paper has been carried out in the frame of these

This work was supported by the European Space Agency and Deimos Enginheria (Portugal) under contract DME-AS-2010-001; and by the Spanish Ministry of Science and Technology and FEDER under projects TEC201125865 and AYA2012-39356-C05-01

I. Corbella, I. Durán, F. Torres, and N. Duffo are with the Remote Sensing Laboratory, Universitat Politècnica de Catalunya, 08034 Barcelona, Spain, and also with the SMOS Barcelona Expert Centre, 08003 Barcelona, Spain (e-mail: \{corbella, israel.duran, xtorres, duffo\} @ tsc.upc.edu.)

$\mathrm{L}$. Wu is with the CAS Key Laboratory of Microwave Remote Sensing from the National Space Science Center (NSSC), Beijing, China. (e-mail: wulin@mirslab.cn)

A. Khazâal is with the Centre d'Etudes Spatiales de la BIOsphère (CESBIO), Toulouse, France. (e-mail: ali.khazaal@cesbio.cnes.fr

M. Martín-Neira is with the European Space Research and Technology Centre, European Space Agency, 2200 AG Noordwijk, The Netherlands (email: Manuel.Martin-Neira@esa.int) activities with the authors' institution (Universitat Politècnica de Catalunya) acting as ESL.

SMOS payload is the Microwave Imaging Radiometer using Aperture Syntyhesis (MIRAS). It consists of a Y-shape interferometric radiometer formed by an array of 69 lowgain dual-polarization antennas placed along the three arms of the structure [4]. Cross-correlation of the signals collected by a pair of receivers provides, after calibration, a sample of the so-called visibility function, in units of Kelvin. This function, in turn, is related to the scene brightness temperature by the visibility equation [5], so an inversion procedure is carried out in order to recover the brightness temperature. In SMOS processing, the offset term due to receiver thermal coupling predicted in [5] is removed by subtracting all science measurements from the visibility of the deep sky -a "flat target" [6]-, which was measured once for this purpose. Discretization of the visibility equation converts it to a system of linear equations, which is solved by standard linear algebra [7] [8] [9]. This process, often referred to as "image reconstruction", is much more involved than at first glance would seem. Antenna pattern differences between elements, antenna characterization uncertainties, calibration errors, aliasing, solar and galactic radiation and other effects produce non-negligible artifacts that have to be mitigated using specific techniques (details can be found for example in [10] and [11]).

Nevertheless, some residual errors persist. One of them, of importance, is called "Land Sea Contamination" (LSC) and consists fundamentally of a slight increase of brightness temperature of water in zones near large land areas. This contamination extends to distances much larger than the relatively low resolution of the instrument would predict. It was reported early in the mission by the SMOS ocean science team to be associated generally with both positive and negative biases, and it has been so far the most important limitation in using SMOS data for coastal areas. It is important for salinity retrieval since the first Stokes parameter varies by only about $1 \mathrm{~K} / \mathrm{psu}$. Part of the LSC can be attributed to the so called floor error [10] and can be slightly reduced by using differential techniques before visibility inversion. However, no image reconstruction method has yet been able to fully cancel this artifact. This paper presents an analysis of the problem, proposes second mechanism that produces LSC, and proposes a method to mitigate this contribution. 


\section{SCALING OF BRIGHTNESS TEMPERATURE ERRORS}

The visibility function (fundamental MIRAS measurement) depends on two variables named $u$ and $v$. For a given antenna pair, they are defined respectively as the relative $(x, y)$ coordinates of one antenna with respect to the other normalized to the center wavelength. The particular case of the origin $u=v=0$ corresponds to both antennas collapsing in a single location. In this case the visibility becomes equal to the antenna noise temperature for this particular element $V(0,0)=T_{A}$. MIRAS uses three redundant Noise Injection Radiometers (NIR) to measure the visibility at zero spacing; and digital correlators to measure the rest of visibility samples. As it will be seen below, this different implementation is important in the assessment of the land-sea contamination problem.

Since the zero-spacing visibility is the average antenna temperature of the scene measured with antennas having a very wide pattern, it has contributions from all the sources in front of the instrument. When the boresight travels from ocean to land, the antenna temperature starts increasing as soon as the main lobe of the antenna pattern reaches the coast. Due to the large antennas beamwidth, it starts when the instrument boresight is still at large distances from the coast. This feature itself would not produce land-sea contamination, since the image reconstruction synthesizes a narrow pattern at each pixel, and those pointing to the ocean would not be affected. Land sea contamination would be observed if an error scaling with antenna temperature is present in the retrieved image. The purpose of the analysis presented below is to demonstrate that this error is present and to find its origin.

Brightness temperature is a function of two variables named $\xi$ and $\eta$ defined as the direction cosines with respect to the instrument reference frame $(x, y)$. It is obtained in the retrieval process as a column vector having as many rows as $(\xi, \eta)$ grid points. Specifically, it is the result of multiplying the pseudo inverse of the G-matrix, $G^{+}$, by the vector of measured visibility samples, including the ones at zero-spacing and the rest. This means that $G^{+}$has as many columns as measured visibility samples in the $(u, v)$ plane and as many rows as $(\xi, \eta)$ grid points. The matrix multiplication $T=G^{+} V$ can then be re-written as:

$$
T=G_{0}^{+} V_{0}+G_{k j}^{+} V_{k j}
$$

where $G_{0}^{+}$is the column of $G^{+}$corresponding to $u=v=0$ and $G_{k j}^{+}$the other columns of the matrix. This equation is useful to introduce errors separately in the zero-spacing visibility $V_{0}=V(0,0)$ and in the other samples $V_{k j}$. Errors should be different because measurement techniques are also different in both cases.

For the following error analysis, the measured magnitudes (represented with a hat) are assumed to be related to the errorfree ones taking into account a constant multiplicative error $\varepsilon$. Three cases are considered:

a) Error affecting only the zero-spacing visibility (antenna temperature). In this case $\hat{V}_{0}=V_{0}(1+\varepsilon)$, which substituted into (1) results in

$$
\hat{T}=G_{0}^{+} V_{0}(1+\varepsilon)+G_{k j}^{+} V_{k j}=T+G_{0}^{+} V_{0} \varepsilon
$$

b) Error present only in the nonzero-spacing visibility samples. In this case, $\hat{V}_{k j}=V_{k j}(1+\varepsilon)$ and the brightness temperature vector (1) becomes

$$
\hat{T}=G_{0}^{+} V_{0}+G_{k j}^{+} V_{k j}(1+\varepsilon)=T(1+\varepsilon)-G_{0}^{+} V_{0} \varepsilon
$$

c) Error in all visibility samples. In this case the separation into two types of visibility samples has no effect. Directly from the general equation:

$$
\hat{T}=G^{+} V(1+\varepsilon)=T(1+\varepsilon)
$$

That is, the retrieved brightness temperature is simply affected by a scale factor with the sole effect of degrading absolute accuracy.

Cases a) and b) show an error term (the second one) that scales with the zero-spacing visibility $V_{0}$, which is the antenna temperature so they will give rise to land-sea contamination error. Note the sign difference of this term in both cases. This error term is also weighted by the zero-spacing column of the inverse G-matrix $G_{0}^{+}$. This is a function of $(\xi, \eta)$ approximately equal to the reciprocal of the power antenna pattern $\left|F_{n}(\xi, \eta)\right|^{2}$ normalized with respect to the antenna solid angle $\Omega_{p}$ and the obliquity factor: ${ }^{1}$

$$
G_{0}^{+}(\xi, \eta) \approx \frac{\Omega_{p} \sqrt{1-\xi^{2}-\eta^{2}}}{\left|F_{n}(\xi, \eta)\right|^{2}}
$$

When drawn as function of $\xi, \eta$, it shows a kind of "bowl shape" being minimum at boresight and growing continuously to the extremes of the field of view. The error terms in both (2) and (3) should inherit this shape.

In conclusion, only cases a) and b) produce land sea contamination due to the presence of an error term depending on the antenna temperature. Contributions from one or other are opposite in sign. Case c) does not yield any land sea contamination. Additionally, cases b) and c) have a multiplicative error at each spatial direction (a scale factor).

\section{Simulation RESUlts}

An end to end simulation using a Fresnel (specular) model of the ocean has been carried out to confirm the theoretical findings. First, an instrument model (G-matrix) is used to compute the expected visibility function; then a multiplicative error is applied either to antenna temperature or to the nonzerospacing visibility samples and finally image reconstruction is performed using the inverse of the G-matrix. No other source of error has been included; in particular the floor error has been eliminated by setting to zero the brightness temperature at points outside the principal hexagon (see [11] for details). Figure 1 shows that a 5\% amplitude error on the zero-spacing baseline -case a)- produces a similar bowl shape spatial error distribution in brightness temperature than a negative $5 \%$ amplitude error only affecting the set of nonzero-spacing visibility samples -case b)-. Although the error distribution is similar in both plots, in the second case the retrieved image has a positive $5 \%$ scale factor [see (3)] that makes the overall scene mean error to be zero. This is consistent with the fact that there is no error in the zero baseline (zero spatial frequency).

\footnotetext{
${ }^{1}$ For an instrument having all antenna patterns identical this is exact
} 

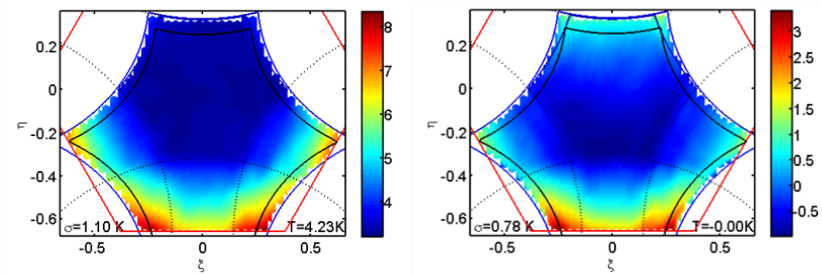

Fig. 1. Simulation of the spatial error (horizontal polarization) over pure Ocean (Fresnel model) due to a 5\% amplitude error on $T_{A}$ (left) and a negative $5 \%$ error on the visibility samples (right). Note that both color bars have the same $4.5 \mathrm{~K}$ range.

The simulations in figure 2 show the predicted error in the first Stokes parameter divided by 2 for a pure (left) and a mixed land-sea (right) snapshot scenes. Ocean is modeled as in the previous simulation and land as constant brightness temperature at $280 \mathrm{~K}$. In this case, a $2 \%$ amplitude error has been artificially included, but no error reduction mechanisms have been applied, so the spatial biases and floor error artifacts are also present. Comparing both images, it is apparent that the error in the ocean part changes in the presence of nearby land masses. The error image at the left is not zero because of the floor error [10], which is the spread of aliasing into the alias-free zone due to having non-identical antenna patterns. In practice, this error can be evaluated and substracted to all measurements in order to produce cleaner images. This technique is commonly used by the SMOS ocean science team to correct data and retrieve salinity with higher accuracy, and it is usually referred to as "OTT ${ }^{2}$ correction" [12]. With this definition, the image at the left of figure 2 is the OTT derived from the pure ocean scene. If it is used to correct the ocean pixels in the mixed scene (Fig. 2, right) the error does not cancel, but becomes proportional to the antenna temperature difference between the pure ocean and the mixed land-sea scene, so producing land-sea contamination.
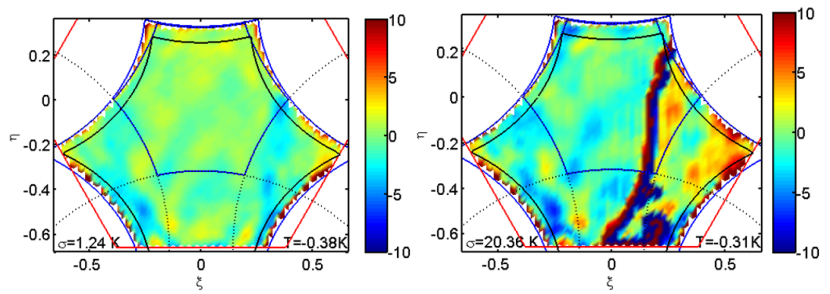

Fig. 2. Simulation of the expected spatial error in the first Stokes parameter divided by 2 due to the combined effect of floor error and a $2 \%$ amplitude error on the visibility samples over ocean (Fresnel model) for a pure Ocean scene (left) and a mixed land-sea scene (right). Land is modeled at a constant $\mathrm{Tb}=280 \mathrm{~K}$. In the mixed scene, LSC is dominated by the contribution from the floor error..

The simulations in Fig. 3 clearly show this effect when a negative $2 \%$ error in the visibility samples other than $V(0,0)$ is taken into account -case b)-. Three snapshots along a descending orbit over the Pacific (leftmost figure) are selected in this example. The pure Fresnel ocean scene in Fig. 2 (left) is selected as reference (OTT) to correct for spatial errors.

${ }^{2}$ Ocean Target Transformation
Obviously, when applied to this reference image, the OTT corrects for all errors (Fig. 3, center-left). However, for mixed land-sea scenes, when the sea pixels are corrected with the OTT the mean error increases as the land masses enter into the single antenna beamwidth (Fig. 3, center-right and rightmost). The bowl shape effect over the ocean is also clearly seen in the last case. The ripple is related to the Gibbs effect due to the sea-land transition. In order to better highlight the impact of the land sea contamination, the floor error has been eliminated in these simulations by setting to zero the brightness temperature of the forward model at points outside the principal hexagon (see [11] for details).

\section{MIRAS CALIBRATION ERRORS}

The visibility sample measured by a pair of MIRAS receivers $k$ and $j$ is computed from the measured magnitudes according to [13]:

$$
V_{k j}=\frac{M_{k j}}{G_{k j}} \sqrt{\frac{v_{k}-v_{o f f k}}{G_{k}} \frac{v_{j}-v_{o f f j}}{G_{j}}}
$$

where the raw measurements are $M_{k j}$, the normalized complex correlation (measured by the digital correlator) and $v_{(k, j)}$, the voltages measured by the Power Measurement System (PMS) implemented in each receiver. The other terms are calibration parameters: $G_{k j}$ the correlator efficiency, and $G_{(k, j)}$ and $v_{\text {off }(k, j)}$ the PMS gains and offsets respectively. During normal operation, the instrument goes periodically into calibration mode to measure and update them [14]. According to section II, in the absence of antenna temperature error, multiplicative errors in $V_{k j}$ contribute to land-sea contamination -case b)-. This can be only produced by PMS gain and/or correlation efficiency calibration errors.

On the other hand, the zero-spacing visibility is the antenna temperature measured by the NIR units. For each one, it is retrieved (neglecting second order corrections) using [15]:

$$
T_{A}=T_{U}-\eta T_{N A}
$$

where $\eta$ is the measured fraction of the Dicke cycle, $T_{U}$ is the internal load physical temperature and $T_{N A}$ the noise diode injected temperature, both of them referred to the antenna reference plane. This last parameter $\left(T_{N A}\right)$ is periodically measured and updated during instrument calibration operation and $T_{U}$ is measured by a thermal sensor. Equations for conversion to antenna reference plane use nominal, on-ground characterized parameters. In the latest version of the L1OP, only one of the three NIRs is used since it shows improved seasonal and long-term stability. In any case, according to section II, land sea contamination is caused by a multiplicative error in $T_{A}$, which can only be present if there is a calibration error of $T_{N A}$. In other words, the calibration error contribution to LSC is a multiplicative error in TA

Amplitude calibration errors on the visibility and on the antenna temperature are decoupled, so both contribute to land sea contamination in a different proportion. It even may happen that an empirical correction in one of them, adding a positive error for example, compensates a negative error 

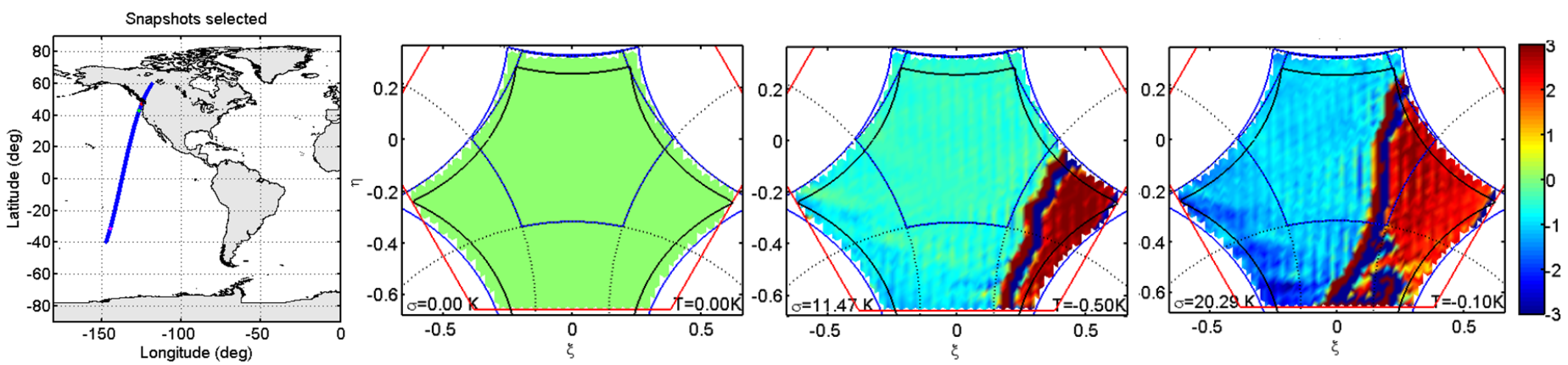

Fig. 3. Simulation of the expected spatial error, after applying the OTT, due to a $2 \%$ error on the visibility samples for three snapshots in a descending orbit (leftmost). The OTT corrects both spatial bias and mean error in the reference snapshot (center-left). As land enters into the single antenna beamwidth, the amplitude error yields an incremental bias proportional to antenna temperature difference with respect to the reference scene that affects the ocean pixels.

in the other, yielding good performance in terms of land-sea contamination but poor calibrated science data.

In order to discard one of them, antenna temperature has been re-computed using the PMS of each of the individual receivers, calibrated by the same gains and offsets as in (6). This approach is implemented in the MIRAS testing software (MTS) [16] and has been called "all-LICEF mode" in reference to the name given to the MIRAS individual receivers: "LIghtWeight Cost-Effective Front-end". In this mode, SMOS data is processed from level 0 in order to bypass the calibration procedures of the L1OP. The zero-spacing visibility is then computed as the mean antenna temperature measured by the 69 power detectors in each single receiver according to the one-point calibration scheme [17]:

$$
T_{A}=\left\langle\frac{v_{k}-v_{\text {off } k}}{G_{k}}-T_{R k}\right\rangle
$$

where $T_{R k}$ is the noise temperature of receiver $k$. Calibration errors on $v_{\text {off } k}$ and $T_{R k}$ are discarded as source of land sea contamination since they produce a scene independent additive error on the antenna temperature that is removed by the OTT.

According to (6) and (8), a common power detector gain calibration error affecting all units, $\hat{G}_{k}=G_{k}(1+\Delta G)$ produces the same amplitude error in both antenna temperature and visibility samples. In consequence, using the receivers antenna temperatures for estimating the zero-spacing visibility should not produce land sea contamination, unless the visibility error comes from the term $G_{k j}$ in (6). Since land sea contamination has been observed in this case, the conclusion is that there is a calibration error affecting the correlator efficiency.

If the amplitude error is related to the correlator efficiency $\hat{G}_{k j}=G_{k j}(1+\Delta G)$, this impacts only the visibility samples measured though correlation, yielding the land sea contamination effect described in (3). In the "All-LICEF mode" this happens to be the only source of land-sea contamination, and this feature has been used to identify a $2 \%$ mean overestimation of correlator efficiency as the source of land sea contamination (see section V).

Correlation efficiency is periodically measured on-board using the procedures defined in [17], but some underlying assumptions, such as dominant RF filter response, I/Q branches similarity or quadrature error correction induce small but relevant uncertainties that degrade the accuracy of the retrieval. As a matter of fact, the measurement accuracy of this parameter has been never validated on ground.

\section{VALIDATION OF SMOS LAND SEA CONTAMINATION MITIGATION}

Figure 4 (top) shows a composite error map (measurement minus model) of the first Stokes parameter divided by two for descending orbits. Data is processed using the "All-LICEF" mode substracting to all snapshots a single OTT computed from a mid Pacific set of consecutive snapshots. The global reference map has been provided by the SMOS ocean science team (see acknowledgment) using the salinity reference of the World Ocean Atlas 2009 [18] and a model similar (but not identical) to that described in [19]. Spatial and time averaging has been applied to yield the final global map (10 days: 1st to 10th May 2011 and 0-60 deg angle of incidence). In this map the land sea contamination effect is clearly seen as a region of warmer brightness temperature surrounding the land masses. However, as shown in the same composite map after applying a $2 \%$ correction on correlator efficiency (Fig 4, bottom), contamination disappears while keeping geophysical features as for example the mid Pacific warmer area (A), the Panama upwelling (B) or the Amazon River Plume (C). Figure 5 plots the difference between the two maps in Fig.4, showing that, as expected, the correlator efficiency correction exclusively affects the areas close to the land masses.

The residual land-sea contamination still seen in figure 4 is due to the floor error contribution, which has not been removed.

\section{CONCLUSION}

Once the major sources of spatial bias in SMOS imagery are successfully mitigated using averaging (spatial and temporal) and enhanced imaging techniques, global error maps still show an anomalous increase of the oceans' brightness temperature near large land masses. This "land-sea contamination" is related to residual multiplicative errors affecting in a different way the visibility at the origin and the other visibility samples. The "all-LICEF" mode improves the calibration consistency between both kinds, reducing the possible differences to only one single calibration parameter: the correlator efficiency $G_{k j}$. A $2 \%$ overestimation of this parameter is the dominant contributor to the observed land-sea contamination. 

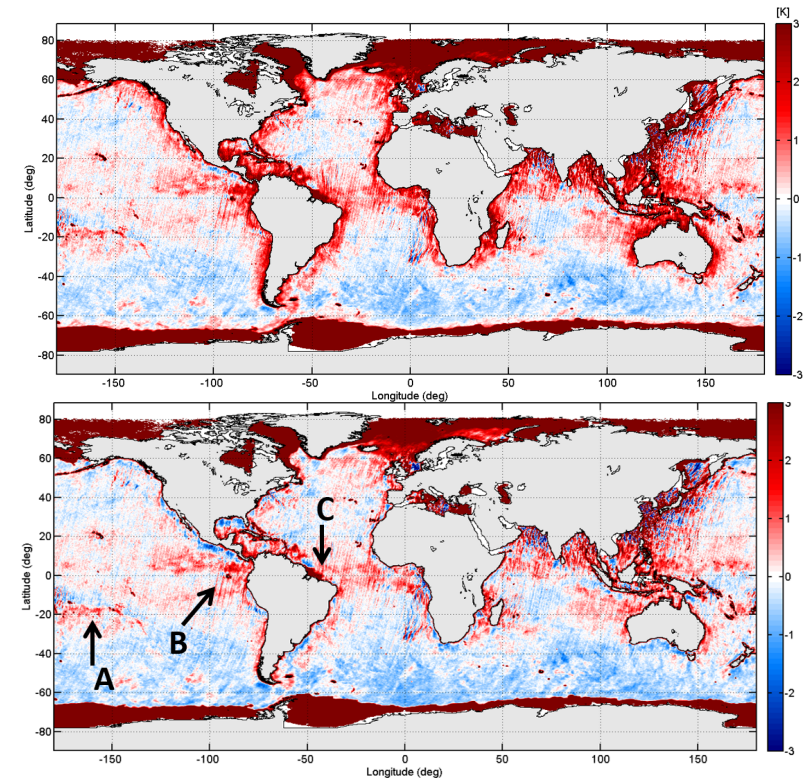

Fig. 4. Global error map of first stokes parameter divided by two for descending orbits over the ocean after applying the OTT before (top) and after (bottom) applying a 2\% correction on the measured correlation efficiency. Averaging: 1st to 10th May 2011 and 0-60 deg incidence angle. Error relative to the ocean model.

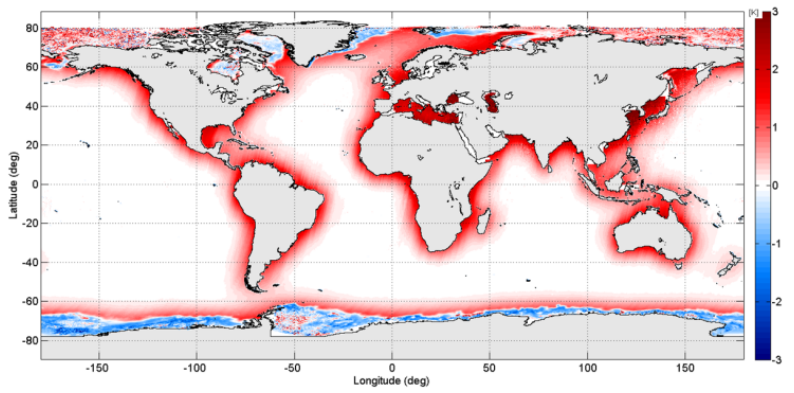

Fig. 5. Difference between the two "all-LICEF" maps in Fig. 4 to show that the $2 \%$ correction on correlation efficiency exclusively affects the regions close to land masses.

Using all-LICEF mode, land sea contamination is insensitive to any antenna temperature error (e.g. due to orbital or seasonal drift) and is corrected only by adjusting the correlator efficiency. This property might consolidate a decision to operate SMOS in "All-LICEF" mode in a future version of the processor. Validation of the correction procedure shows how complementary calibration routines together with image synthesis autoconsistency properties provides a very robust SMOS performance.

\section{ACKNOWLEDGMENT}

The authors would like to thank Joseph Tenerelli (formerly in CLS, now in OceanDataLab, France) for providing the ocean model.

\section{REFERENCES}

[1] H. Barré, B. Duesmann, and Y. Kerr, "SMOS: The mission and the system," IEEE Transactions on Geoscience and Remote Sensing, vol. 46, no. 3, pp. 587-593, March 2008.
[2] S. Mecklenburg, M. Drusch, Y. H. Kerr, J. Font, M. Martín-Neira, S. Delwart, G. Buenadicha, N. Reul, E. Daganzo-Eusebio, R. Oliva, and R. Crapolicchio, "ESA's soil moisture and ocean salinity mission: Mission performance and operations," IEEE Transactions on Geoscience and Remote Sensing, vol. 50, no. 5, pp. 1354-1365, May 2012.

[3] L. Wu, F. Torres, I. Corbella, N. Duffo, I. Durán, M. Vall-llossera, A. Camps, S. Delwart, and M. Martín-Neira, "Radiometric performance of SMOS full polarimetric imaging," IEEE Geoscience and Remote Sensing Letters, vol. 10, no. 6, pp. 1454-1458, November 2013.

[4] K. McMullan, M. Brown, M. Martín-Neira, W. Rits, S. Ekholm, J. Marti, and J. Lemanzyk, "SMOS: The payload," IEEE Transactions on Geoscience and Remote Sensing, vol. 46, no. 3, pp. 594-605, March 2008.

[5] I. Corbella, N. Duffo, M. Vall-llossera, A. Camps, and F. Torres, "The visibility function in interferometric aperture synthesis radiometry," IEEE Transactions on Geoscience and Remote Sensing, vol. 42, no. 8 , pp. 1677-1682, August 2004.

[6] M. Martín-Neira, M. Suess, and J. Kainulainen, "The flat target transformation," IEEE Transactions on Geoscience and Remote Sensing, vol. 46, no. 3, pp. 613-620, March 2008.

[7] A. Camps, M. Vall-llossera, I. Corbella, N. Duffo, and F. Torres, "Improved image reconstruction algorithms for aperture synthesis radiometers," IEEE Transactions on Geoscience and Remote Sensing, vol. 46, no. 1, pp. 146-158, January 2008.

[8] E. Anterrieu and A. Khazaal, "Brightness temperature map reconstruction from dual-polarimetric visibilities in synthetic aperture imaging radiometry," IEEE Transactions on Geoscience and Remote Sensing, vol. 46, no. 3, pp. 606-612, March 2008.

[9] I. Corbella, F. Torres, A. Camps, N. Duffo, and M. Vall-llossera, "Brightness temperature retrieval methods in synthetic aperture radiometers," IEEE Transactions on Geoscience and Remote Sensing, vol. 47, no. 1, pp. 285-294, January 2009.

[10] I. Corbella, F. Torres, L. Wu, N. Duffo, I. Duran, and M. Martín-Neira, "Spatial biases analysis and mitigation methods in SMOS images," in International Geoscience and Remote Sensing Symposium, IGARSS 2013. Melbourne, Australia: IEEE, 21-26 July 2013, pp. 3145-3418.

[11] - "SMOS image reconstruction quality assessment," in International Geoscience and Remote Sensing Symposium, IGARSS 2014. Québec, Canada: IEEE, 13-18 July 2014, pp. 1914-1916.

[12] J. Font, J. Boutin, N. Reul, P. Spurgeon, and others ..., "SMOS objectives and approach for ocean salinity observations," in ESA Living Planet Symposium, E. S. A. (ESA), Ed., vol. SP-686, Bergen, Norway, 28 June -2 July 2010

[13] I. Corbella, F. Torres, A. Camps, A. Colliander, M. Martín-Neira, S. Ribó, K. Rautiainen, N. Duffo, and M. Vall-llossera, "MIRAS end-toend calibration. Application to SMOS L1 processor," IEEE Transactions on Geoscience and Remote Sensing, vol. 43, no. 5, pp. 1126-1134, May 2005.

[14] M. Brown, F. Torres, I. Corbella, and A. Colliander, "SMOS calibration," IEEE Transactions on Geoscience and Remote Sensing, vol. 46, no. 3, pp. 646-658, March 2008 .

[15] A. Colliander, L. Ruokokoski, J. Suomela, K. Veijola, J. Kettunen, V. Kangas, A. Aalto, M. Levander, H. Greus, M. T. Hallikainen, and J. Lahtinen, "Development and calibration of SMOS reference radiometer," IEEE Transactions on Geoscience and Remote Sensing, vol. 45, no. 7, pp. 1967-1977, July 2007.

[16] I. Corbella, F. Torres, N. Duffo, V. González, A. Camps, and M. Vallllossera, "Fast processing tool for SMOS data," in International Geoscience and Remote Sensing Symposium, IGARSS 2008, no. II. Boston(Ma), USA: IEEE, 7 - 11 July 2008, pp. 1152-1155.

[17] F. Torres, I. Corbella, A. Camps, N. Duffo, M. Vall-llossera, S. Beraza, C. Gutierrez, and M. Martín-Neira, "Denormalization of visibilities for in-orbit calibration of interferometric radiometers," IEEE Transactions on Geoscience and Remote Sensing, vol. 44, no. 10, pp. 2679-2686, October 2006.

[18] J. I. Antonov, D. Seidov, T. P. Boyer, R. A. Locarnini, A. V. Mishonov, H. E. Garcia, O. K. Baranova, M. M. Zweng, and D. R. Johnson, World Ocean Atlas 2009 Volume 2: Salinity, S. Levitus, Ed., NOAA Atlas NESDIS 69 U.S. Government Printing Office, Washington, D.C. 184 pp., March 2010.

[19] S. Zine, J. Boutin, J. Font, N. Reul, P. Waldteufel, C. Gabarró, J. Tenerelli, F. Petitcolin, J.-L. Vergely, M. Talone, and S. Delwart, "Overview of the SMOS sea surface salinity prototype processor," IEEE Transactions on Geoscience and Remote Sensing, vol. 46, no. 3, pp. 621-645, March 2008. 